\title{
Significado del cuidado de la gestante desde la experiencia de la enfermera
}

\author{
Meaning of pregnant care from nurse's experience \\ Nayibe Guerra ${ }^{1}$, Rosa Reina ${ }^{2}$, María Hilda Cárdenas ${ }^{2}$, Flor Sanmiguel ${ }^{2}$
}

\section{RESUMEN}

Objetivo: Se plantea interpretar el significado del cuidado de la gestante desde la experiencia de la enfermera. Materiales y Métodos: la investigación es cualitativa, fenomenológica hermenéutica. La técnica de recolección de información fue la entrevista a profundidad, mediante encuentros directos con los 3 actores claves. Para el desarrollo de este estudio y el análisis de datos fue el método de Spiegelberg que permite estudiar el campo de la conciencia y explorar a las personas en estudio, partiendo de sus vivencias y experiencia cuyos pasos están constituidos por fases. Resultados: Del análisis de las entrevistas surgieron 2 Categorías: Acciones de enfermería sentido de compromiso y Preocupación, factores de riesgos más frecuentes. De aquí se puede deducir que el cuidado se ha convertido en la esencia de enfermería evidenciada desde el transcurso de la historia. Conclusiones: la intervención de enfermería en la consulta prenatal; tiene que ir más allá del protocolo institucional; donde la mujer en su condición de embarazo es vista como una unidad de atención donde se promociona su salud y la de su hijo y se previenen complicaciones; de tal manera que el cuidado humano dado por la enfermera hacia la gestante en la consulta prenatal debe demostrar interés por la persona, el estar allí y comunicarse de manera empática, que favorezca relaciones de reciprocidad y muestra de interés por las necesidades presentes y sentidas de la embarazada, debe existir un compromiso de apoyo, solidaridad y respeto por la condición de la persona que le presta ese cuidado humano.

Palabras claves: significado, cuidado, gestante, experiencia, enfermera

\begin{abstract}
Objective: To interpret the meaning of the care of the pregnant woman from the experience of the nurse. Materials and Methods: research is qualitative, hermeneutical phenomenological. The technique of gathering information was the in-depth interview, through direct meetings with the 3 key actors. For the development of this study and data analysis was the Spiegelberg method that allows to study the field of consciousness and explore the people in study, based on their experiences and experiences whose steps are constituted by phases. Results: Two categories emerged from the analysis of the interviews: Nursing actions sense of commitment and concern, more frequent risk factors. From here it can be deduced that care has become the essence of nursing evidenced from the course of history. Conclusions: the nursing intervention in the prenatal consultation; it has to go beyond the institutional protocol; where the woman in her pregnancy condition is seen as a unit of attention where her health and that of her child is promoted and complications are prevented; in such a way that the human care given by the nurse to the pregnant woman in the prenatal consultation must show interest in the person, be there and communicate in an empathic way, favoring reciprocal relationships and showing interest in the present and felt needs of the patient. the pregnant woman, there must be a commitment of support, solidarity and respect for the condition of the person who gives her that human care.
\end{abstract}

Keywords: meaning, care, gestant, experience, nurse

${ }^{1}$ Coordinación Red Atención Primaria ASIC Campo de Carabobo Municipio Libertador Edo. Carabobo-Venezuela. ${ }^{1}$ Universidad de Carabobo. Facultad de Ciencias de la Salud. Escuela de Enfermería. Venezuela.

\section{INTRODUCCIÓN}

El cuidado se ha convertido en la esencia de enfermería evidenciada desde el transcurso de la historia, donde se han generado reflexiones y ha sido estudiado desde diferentes puntos teóricos y científicos con perspectivas en los paradigmas en las expresiones del cuidar que se van modificando en el transcurso del tiempo. De igual manera se ha visto el cuidado influenciado en los cambios y 
fenómenos sociales, culturales, políticos y ambientales que modifican la conducta en el ser humano y en el modo de vida, desde sus orígenes el ser humano a requerido cuidados y atención para mantener su salud y en la actualidad sigue siendo la misma necesidad por lo cual el cuidar y el cuidado son conceptos que identifican a enfermería, destacando su importancia como fundamento en la práctica para preservar la vida y dignidad humana.

El ser humano es subjetivo en sus apreciaciones, distintas corrientes filosóficas han dado respuestas que el hombre o ser humano es la unión de un cuerpo material y alma espiritual. (1) Por lo tanto el ser humano comprende, razona y requiere conforme a un principio objetivo comprendido y libremente aceptado por el mismo. Es decir el ser humano descubre en sí y por sí mismo que su fin es el de constituirse como persona y que está orientado a un fin superior de forma de unidad con vida espiritual y armónica, basándose en el cuidado desde sus inicios.

Considerando el ser humano desde este enfoque surge el concepto de cuidado integral hacia el individuo dado como respuesta que el profesional de enfermería debe de estar centrado en las necesidades humanas fundamentales del proceso de desarrollo que a su vez busca como finalidad el bienestar individual, sobre todo cuando es un embarazo. De allí que el cuidar y el cuidado son conceptos que identifican la disciplina de enfermería ambos ligados profundamente al ser humano, ninguna especie sobrevive sin cuidado, este cuidado ha sido conceptualizado de diferentes maneras y ha sido objeto de múltiples discusiones, reflexiones, investigaciones, y conferencias. (1)

El cuidado humano se ha convertido en la esencia de enfermería evidenciado por ser una invariante histórica, que ha generado reflexiones discusiones y ha sido estudiado desde diferentes perspectiva. En la enfermería el cuidado es imperativo para promover la salud y la calidad de vida de cada ser. (2) Es decir mediante la transformación la práctica del cuidado de enfermería a través de los años se orienta a la disminución de enfermedades mediante la promoción y prevención el cual se ha visto influenciado por los cambios sociales, económicos y culturales de las gestantes.

Por su parte, el cuidado según Rodríguez, (2) es un acto de vida, una responsabilidad individual que pasa a ser un acto profesional cuando la persona humana presenta diferencias relacionadas con la salud, en habilidades para su autocuidado, en conocimientos en la disposición de cuidados humanos y ambientales para mantener la salud. Es un proceso de interacción dinámico, un compromiso de apoyo, un acompañamiento presente en todas las actividades de la vida. (2) De este modo solo se cuida lo que se valora, enfermería afianza sus conocimientos utilizando diferentes abordajes en el cuidado a las gestantes en el control prenatal afianzando las teorías y modelos relacionados con la promoción de salud y su mantenimiento.

Según Watson, refiere la prestación del cuidado no es solo una emoción, preocupación, actitud o deseo benevolente. Cuidar connota una respuesta personal, la prestación humana de cuidados supone valores una voluntad $\mathrm{y}$ compromiso con el cuidado, conocimiento, acciones de cuidar y consecuencias. El ideal y el valor del cuidar es un punto de arranque, la característica más abstracta de una persona que cuida es que de alguna manera responde a la persona que cuida como individuo único. (3) En este sentido la enfermería tiene como finalidad el cuidado de la salud de la gestante (binomio madre e hijo), por lo que enfoca sus acciones en investigar y analizar las causas reales que ocasionan riesgos durante su gestación por eso es importante que acuden al control.

Cada vez que una mujer se embaraza, existe una probabilidad de que ella o su futuro hijo sufran un fenómeno adverso durante el proceso reproductivo. Esto se conoce como riesgo reproductivo. (4) Históricamente se ha intentado reducir el mismo a través del control prenatal de calidad, la atención del parto por personal capacitado y los cuidados durante el puerperio. A pesar de que aun en los centros de salud se está lejos de universalizar estas prestaciones con la calidad suficiente, se debe comenzar a emplear en la atención de control disminuyendo el riesgo reproductivo. Así mismo Salazar, afirma que, una vez percibida la importancia de la vida fetal, se debería cuidar de la salud de la gestante, no solo física, sino también mental. (4) Vale la pena añadir que después de que la implicación emocional de las consecuencias de un embarazo, es necesario cuidar la salud mental de la pareja que la vive. Esto implica deberes para los profesionales del área de salud.

Además el embarazo es un estado de salud normal, un estado saludable por el que las mujeres en su mayoría aspiran o pasan en algún momento de su vida, bien sea por planificación o por circunstancias que en su vida no fueran deseadas. (4) Sin embargo, este es un proceso donde se pueden entrañar graves riegos que pudieran conllevar complicaciones derivadas por el embarazo como la muerte o discapacidad, no obstante, la mayoría podría ser evitada con las 
medidas preventivas de cuidados adecuados por el equipo de salud en el control prenatal.

Del mismo modo la consulta prenatal forma parte de esta adaptación, donde la enfermera cumple un rol importante, pues su responsabilidad atender a la gestante en un ambiente de comodidad, y utilizando una comunicación abierta en cada valoración prenatal, por lo cual la enfermera debe centrar su interés en la mujer como un binomio, valorando su estado físico y psicológico, lo cual repercute en el feto y su crecimiento y desarrollo hasta llevar su embarazo a término. (4) Sin embargo durante el control prenatal se aplican unas algunas medidas que contribuyen a que el embarazo transcurra $y$ culmine exitosamente.

A sí mismo el control prenatal se considera como la piedra angular que permite evitar $y$ controlar la morbilidad y los causales de la muerte perinatal fundamentándose en la detección y prevención oportuna de los problemas de salud, siendo la evaluación de su efectividad complicada por la presencia de factores de riesgos. El control prenatal es uno de los elementos indispensable para prevenir y en lo posible tratar, patologías maternas que pueden afectar el desarrollo y condición del feto, así como patologías propias de éste que puedan condicionar morbimortalidad materna y perinatal (4).

Por otra parte, el cuidado prestado por enfermería a la gestante debería ser eficiente en todos los centros de salud el cual deben garantizar la continuidad del cuidado de la gestante y grupo familiar. La atención a la gestante por enfermería obliga a aplicar enfoques sistémicos y coherentes a la gestión de la problemática de cada una por separado y su continuidad de control sucesivos que le garanticen una ruta de maternidad segura.

Así mismo, las prestaciones de servicios en los controles de las gestantes, el cuidado se enfoca en los cambios necesarios para su atención en los centros de salud de Atención Primaria de Salud (APS) el cual se responsabilizan de una determinada población y sean capaces de coordinar el apoyo brindado por los hospitales con cuidados y atención especializadas. Hoy en día el cuidado que brinda enfermería en el control prenatal a nivel mundial sobre todo en los que respecta a los países desarrollados cumple muy bien con las exigencias de la Organización Mundial de la Salud (OMS), la cual monitorea que se cumplan las exigencias en la manera que habitualmente se implementan los cuidados (5).

Además, las estrategias antes indicadas, se encuentran enmarcadas en el cumplimiento de los Objetivos de Desarrollo del Milenio (ODM) en su Meta 5: "Reducir, entre 1990 y 2015, la mortalidad materna en tres cuartas partes y lograr, para el año 2015, al acceso universal a la salud reproductiva" (ONU, 2000). Cada año alrededor de 8 millones de mujeres cumplen complicaciones durante el embarazo y de ellas fallecen más de medio millón en la región Subsahariana. Según estimaciones de la OMS, el UNICEF, el UNEFPA, el Grupo del Banco Mundial y la División de Población de las Naciones Unidas, el número anual de muertes maternas disminuyó en un $43 \%$, de aproximadamente 532.000 en 1990 a una cifra estimada de 303.000 en 2015. Las mayorías de estas muertes son evitables aun con recursos limitados pero para ello se necesita dispones de la información adecuada que le permita pasar a los hechos (6).

No obstante, se calcula que a nivel mundial la tasa de mortalidad materna, que es una medida del riesgo obstétrico relacionado con cada embarazo, asciende a 400 por cada 100000 nacidos vivos. Actualmente mediante Objetivos 3 del Desarrollo Sostenible relacionados con la salud, "Garantizar una vida sana y promover el bienestar para todos en todas las edades" enmarca para 2030, reducir la tasa mundial de mortalidad materna a menos de 70 por cada 100.000 nacidos vivos. En los lugares con una elevada tasa de fecundidad, las mujeres se ven enfrentadas a ese riesgo varias veces durante su vida y la probabilidad de morir durante el embarazo o el parto a lo largo de la vida puede ser de hasta 1 entre 16, en comparación con 1 entre 2800 en los países desarrollados. La mortalidad materna es difícil de medir de manera fiable en la mayoría de los países en desarrollo, donde no se registran sistemáticamente las defunciones ni se expiden certificados. Por ese motivo, los datos sobre las tendencias de las tasas de mortalidad materna son escasos (7).

Según el Plan decenal de salud pública (20122021) (MPSP/OMS, 2013), la situación actual de causas de mortalidad materna muestra que el $40 \%$ se debe a afecciones obstétricas no clasificadas; el $21 \%$ a edema, proteinuria y trastornos hipertensivos en el embarazo, el parto y el puerperio; y el $17 \%$ a complicaciones del trabajo de parto y del parto mismo. La mayoría de muertes maternas que se consideran evitables se asocian con deficiencias en el acceso a servicios de salud de calidad, dado que más del $95 \%$ de los partos son institucionales y el $92 \%$ de las mujeres recibieron atención prenatal de un profesional médico. La mortalidad infantil, por su parte, muestra una tendencia al descenso sostenido y una concentración creciente en la etapa neonatal, con el $63 \%$ del total de muertes de menores de un año (8). 
El acceso a la atención de salud reproductiva es la base de la salud materna y en la actualidad no se tienen acceso a los servicios que ofrezcan anticonceptivos, planificación familiar y controles prenatales seguros y eficaces. La asistencia del personal de enfermería es esencial para contribuir a la reducción de la mortalidad materna infantil, ya que como personal calificado debe de garantizar el cuidado humanizado disminuyendo los riesgos obstétricos en las embarazadas.

Cabe destacar, que en la Parroquia Independencia del Municipio Libertador, la red del Área de Salud Integral Comunitaria (ASIC) Campo de Carabobo, en los controles de las gestantes en los Ambulatorios y Consultorios Populares Tipo II, no se están garantizando el continuo del servicio de controles prenatales y el desarrollo de una cultura de cuidados de calidad entre la gestante y el profesional de enfermería. Las gestantes están en inconformidad con la atención en los accesos de los servicios de estas consultas, manifiestan las gestantes y están en inconformidad con la atención falta de información, polivitamínicos, realización de exámenes, ecografías y la dificultad de inclusión en los centros hospitalarios cuando les llega el momento de dar a luz.

Sin embargo, las embarazadas cuando acude al centro de salud de atención primaria esperan ser atendida con calidez, manifiesta temor a los diagnósticos negativos, incomodidad a los procedimientos ginecológicos, evasión de información sobre los cuidados que deben de tener durante su embarazo bien sea por factores económicos o sociales. Las actividades que realiza enfermería de controlar peso, talla, tensión arterial son la que se observan a menudo en toda consulta, dejando atrás las actividades propias de cuidado, el conocimiento del cuidado humanizado y educacional en el control prenatal.

Esta realidad me ha hecho analizar y enfocar el objetivo de esta investigación, abordando el fenómeno de estudio de acuerdo al área de interés anterior descrito se dará dirección a lo planteado en la búsqueda que se pueda responder a la siguiente interrogante: ¿Qué significado le otorga la enfermera al cuidado de la mujer en la consulta prenatal en la red de atención primaria en salud? Para dar respuesta a esta interrogante decidí realizar un estudio con un enfoque cualitativo con abordaje fenomenológico hermenéutico con fin de comprensión que me permita interpretar los conocimientos de las enfermeras en relación del buen control de la gestante en la consulta prenatal. Como objetivo de investigación se plantea interpretar el significado del cuidado de la gestante desde la experiencia de la enfermera.
Dentro del estado del arte se tiene que, Hernández y Vásquez realizaron una investigación en el 2015 en Colombia, El cuidado de Enfermería comprometido: Motor en la satisfacción de la gestante durante el control prenatal. El objetivo de estudio fue describir la percepción acerca de la satisfacción expresada por las gestantes en relación con el cuidado de enfermería, recibido durante el control prenatal La metodología utilizada fue la fenomenología interpretativa y como técnica de recolección de la información se empleó la entrevista a profundidad; la muestra se determinó por saturación de la información; se entrevistaron siete gestantes clasificadas de bajo riesgo obstétrico que asistían regularmente a control prenatal. A cada una se le realizaron dos entrevistas las cuales fueron transcritas por las investigadoras. Y como resultados se identificaron dos temas: "el cuidado de enfermería comprometido como generador de sentimientos de aceptación en el control prenatal" y "la gestante protagonista de su cuidado desea una interacción con la enfermera aún más profunda". Fue posible determinar que la gestante se sintió apoyada y acogida por parte del profesional, que les permitió crecer como seres humanos. (9) Tiene relevancia con la presente investigación ya que el cuidado impartido por enfermería hacia las gestantes debe integrarse y socializarse involucrar al núcleo familiar en las consultas de atención primaria que estén en interacción que se sientan seguras y protagonistas. Así mismo se relaciona con el método utilizado.

Asimismo, Vasconcelos y otros en el 2014, en Brasil, realizaron una investigación titulada: La calidad de asistencia enfermería en el periodo prenatal desde la perspectiva de la mujer embarazada, el objetivo de estudio fue describir la visión de las embarazadas acerca de la calidad de la asistencia de enfermería en el periodo prenatal e identificar las expectativas y necesidades de las mujeres en esa perspectiva. La metodología estuvo enmarcada en una investigación cualitativa, exploratoria, realizada en el Hospital de Rio de Janeiro, Brasil. Participaron veinte embarazadas. Se utilizó la entrevista abierta e individual; el referencial metodológico fue el análisis del contenido de Bardin. La investigación fue aprobada por el Comité de Ética, los sujetos fueron las mujeres embarazadas en su mayoría casadas y con la enseñanza media completa. Como resultados satisfactorios emergieron aquellos referentes por la atención recibida por parte de los enfermeros en el prenatal, mientras la insatisfacción se dio por la infraestructura, los recursos materiales y la falta de una unidad materna para la atención del parto. (10) 
La presente investigación tiene relevancia con el presente estudio, porque enfermería considera que las gestantes cuando han tenido más de dos embarazos poseen el conocimiento para su cuidado, se dejan llevar por creencias que la madre debe de tener conocimientos de su cuidado, cuando estás en su mayoría no ha acudido a control continuo y hay mala desinformación de los riesgos que puedan presentar a la hora del parto. Por lo tanto enfermería debe estar en conocimiento y empatía con la gestante

\section{MATERIALES Y MÉTODOS}

La investigación está dentro del paradigma cualitativo, con un enfoque fenomenológico hermenéutico, por cuanto permite al profesional de enfermería considere tener la calidez humana, brindar amor, confianza, comprensión, cariño y solidaridad hacia el cuidar de las gestantes así como interpretar el significado desde su propia subjetividad, dar cuidado mediante conocimiento, respeto ayudando a la embarazada a mantener su estado de salud, comunicarle la importancia del control. Como técnica de recolección de información que se emplea es la entrevista a profundidad, mediante encuentros directos con los 3 actores claves. Para el desarrollo de este estudio y el análisis de datos fue el método de Spiegelberg que permite estudiar el campo de la conciencia y explorar a las personas en estudio, partiendo de sus vivencias y experiencia cuyos pasos están constituidos por fases.

\section{RESULTADOS Y DISCUSIÓN}

Del análisis de las entrevistas surgieron 2 categorías:

\section{Cuidado en la consulta prenatal}

Orquídea: El cuidado que se le da a un niño, preservan la vida de un ser en su vientre, cuidado debe ser de valor.

Violeta: Lleva una vida en su vientre así que el cuidado es doble, orientación, entender su compromiso, dar comprensión, tener paciencia, escuchar sus inquietudes.

Jazmín: Es de manera preventiva, herramienta, Proceso de atención en cuanto al cuidado se refiere, mejores beneficios.

\section{Control prenatal}

Orquídea: Son actividades que se dan en la consulta a toda gestante, seguimiento cómo evoluciona durante su embarazo.
Violeta: Son actividades que se realizan a diario en la consulta.

Jazmín: Son acciones encaminadas al control de las embarazadas con el objetivo de lograr una mejor atención.

Según los Protocolos de Atención en Cuidados Prenatales y Atención Obstetrica de Emergencia (2014): La consulta prenatal, es un conjunto de acciones médicas y asistenciales que se concretan en entrevistas o visitas programadas con el equipo de salud, a fin de controlar la evolución del embarazo y obtener una adecuada preparación para el parto y la crianza del recién nacido con la finalidad de disminuir los riesgos de este proceso fisiológico.

Desde este punto de vista, se considera que la vivencia tanto de la enfermera como la de las gestantes se manifiestan como una rutina de actividades describiendo satisfacción personal por parte de la enfermera por las actividades que realiza dejando de lado el sentir de la gestante que está a la espera de esa atención de enfermería.

\section{Interpretación del Fenómeno}

En esta fase, se organizaron los datos y se realizaron las interpretaciones sobre el significado que le otorga enfermería al cuidado de la gestante en el control prenatal en la atención primaria de salud. La intervención de enfermería en la consulta prenatal; tiene que ir acrecentándose en las necesidades de las usuarias e ir más allá del protocolo institucional; de tal manera que el cuidado humano dado por la enfermera hacia la gestante en la consulta prenatal debe demostrar interés por la persona, el estar allí y comunicarse de manera empática, que favorezca relaciones de reciprocidad y muestra de interés por las necesidades presentes y sentidas de la embarazada, debe existir un compromiso de apoyo, solidaridad y respeto por la condición de la persona que le presta ese cuidado humano.

\section{CONCLUSIONES}

Descubrir los significados que tiene enfermería sobre el embarazo y la maternidad en general, donde la intervención de enfermería en la consulta prenatal; tiene que ir más allá del protocolo institucional; donde la mujer en su condición de embarazo es vista como una unidad de atención donde se promociona su salud y la de su hijo y se previenen complicación que dependiendo de sus condiciones biológicas, sociales, culturales la pudieran colocar en situación de riesgo para ella o el producto de la 
concepción; de tal manera que el cuidado humano dado por la enfermera hacia la gestante en la consulta prenatal debe demostrar interés por la persona, el estar allí y comunicarse de manera empática, que favorezca relaciones de reciprocidad y muestra de interés por las necesidades presentes y sentidas de la embarazada, debe existir un compromiso de apoyo, solidaridad y respeto por la condición de la persona que le presta ese cuidado humano.

\section{REFERENCIAS BIBLIOGRÁFICAS}

1. Universidad de Carabobo, Direccion de Postgrado. I Congreso Internacional II Congreso Nacional en Cuidado Humano en Memoria de la Dra. Rosa Gonzalez de Gelvez. Valencia: Delforn, C.A, 2009. Vol. I. 978-980-233-502-2.

2. Rodriguez, Dra. Maria Yolanda. El Cuidado Humano y su Impacto en un Mundo Globalizado. Venezuela: Delforn, C.A., 2009. págs. 54-56. 978-980-233-502-2.

3. Martinez, Maria, Ledesma, Maria y Perez, Evangelina. Componente de Formacion Especifica del Preofesional de Enfermeria. Miranda: Bauxe, 2012. EFBE318.

4. Diaz, Inés. Significado que otorga la enfermera a la valoracion de la gestante de alto riesgo en la consulta prenatal. 25 de Enero de 2012.

5. Castaño, Katerinne. Calidad de Atencion en el Control Prenatal, Opinon de las Usuariasen una institucion Prestadora de Servicios de Bogotá. Bogotá : D.C. Colombia, 2013. 978-92-75-32980-1.

6. (OMS), Organizacion Mundial de la Salud. Mas Alla de las Cifras. Enero de 2004. 97892-75-32980-1.

7. Dodd, Rebecca. Organización Mundial de la Salud La salud y los Objetivos de Desarrollo del Milenio. Mantengamos la promesa. Argentina - Diseño y maquetación: Kaolis/Montpellier-Francia. : Ediciones de la OMS, 2017. Vols. Ilustraciones: OMS/Virot P. - OMS/TBP/Davenport, Falise, Hampton, Van der Hombergh, Colors magazine/Langvad J., Clasificación NLM: WA 530.1. ISBN 9243562983.

8. Ruiz, Carmen, y otros. Cuidado de la Mujer Gestante. Bogotá: ACOFAEN, 2014. 978958-8735-68-9.

9. Hernandez, Ana M y Vasquez, Marta L. El Cuidado de Enfermeria Conprometido: Motor de la Satisfaccion de la Gestate en el Control Prenatal. Colombia: Universidad y
Salud, 2015. Rev Univ. salud. 2015;17(1):80-96.

10. Daniel, Suáres. Percepción del Cuidado de Enfermería de las Gestantes que Asisten al Curso Maternidad y Paternidad un Proyecto de Vida. Colombia - Bogóta: Universidad Nacional de Colombia Facultad de Enfermeria, 2014. 